\title{
Laser-induced forward transfer of single-walled carbon nanotubes
}

\section{Journal Article}

\section{Author(s):}

Palla-Papavlu, Alexandra; Dinescu, Maria; Wokaun, Alexander; Lippert, Thomas (D)

Publication date:

2014-10

Permanent link:

https://doi.org/10.3929/ethz-b-000090630

\section{Rights / license:}

In Copyright - Non-Commercial Use Permitted

Originally published in:

Applied Physics A 117(1), https://doi.org/10.1007/s00339-014-8473-5 


\title{
Laser-induced forward transfer of single-walled carbon nanotubes
}

\author{
A. Palla-Papavlu $\cdot$ M. Dinescu • \\ A. Wokaun $\cdot$ T. Lippert
}

Received: 4 November 2013/Accepted: 1 May 2014/Published online: 21 May 2014

(c) Springer-Verlag Berlin Heidelberg 2014

\begin{abstract}
The objective of this work is the application of laser-induced forward transfer (LIFT) for the fabrication of chemiresistor sensors. The receiver substrate is an array with metal electrodes and the active materials placed by LIFT are single-walled carbon nanotubes (SWCNT). The functionality of such sensors depends on the geometry of the active material onto the metallic electrodes. First the best geometry for the sensing materials and electrodes was determined, including the optimization of the process parameters for printing uniform pixels of SWCNT onto the sensor electrodes. The sensors were characterized in terms of their sensing characteristics, i.e., upon exposure to ammonia, proving the feasibility of LIFT.
\end{abstract}

\section{Introduction}

Air pollution is an important issue due to technological developments and rapid urbanization [1]. Gaseous pollutants include sulfur compounds, carbon monoxide, nitrogen compounds, ammonia and organic compounds such as hydrocarbons or odorous substances.

One promising solution for the detection of small quantities of chemical and polluting agents is the application of a new generation of low power, low cost, portable sensing devices for monitoring of manufacturing environments. A very promising candidate is a chemical sensor

A. Palla-Papavlu · A. Wokaun · T. Lippert

General Energy Research Department, Paul Scherrer Institute, PSI, 5232 Villigen, Switzerland

A. Palla-Papavlu $(\varangle) \cdot$ M. Dinescu

National Institute for Lasers, Plasma and Radiation Physics, Magurele, P.O. Box MG-36, RO-077125 Bucharest, Romania e-mail: apalla@nipne.ro based on the change in resistance in response to binding of analytes (a chemiresistor). This type of sensors is very attractive due to low power consumption and very precise readings may be achieved with minimal electronics. Several types of materials have been used as active material in gas sensors, including metal oxides [2], organic semiconductors, and carbon nanotubes (CNT) [3].

Single-walled carbon nanotubes (SWCNT) are promising chemical-sensing materials, due to their hollow structure, one-dimensional nanoscale morphology, and highspecific surface area that benefits physical adsorption or chemical reaction with target molecules for signal transformations with higher efficiency and speed [4-6]. The applicability of devices based on SWCNT systems has been limited by the complexity associated with the device fabrication.

Laser-induced forward transfer (LIFT) has emerged over the last years as a powerful technique for printing a wide class of materials, i.e., inorganic inks and pastes, metals, oxides, polymers and biological materials in solid or liquid phase [7-15]. In addition, CNT have been transferred by LIFT as microarrays which work as field emission cathode devices, and more recently multiwalled CNT dispersed in different polymer matrices were reported [16, 17]. This technique represents an interesting alternative to other printing techniques, since it is free from clogging problems, which are characteristic for inkjet printing, and LIFT avoids the use of expensive photolithographic masks. LIFT presents also a higher potential for integration than another possible deposition methods, e.g., microspotting. In LIFT, the material is transferred by the laser beam from a transparent support or donor onto an appropriate substrate or receiver. For improving the process efficiency and reducing the risk of damaging the layer to be transferred, the donor substrate can be previously coated with a 
polymeric layer, which is called sacrificial layer, dynamic release layer (DRL) or blister actuated [18-22]. The implementation of triazene polymer (TP)-assisted LIFT as a new direct printing laser technique allows to overcome the main drawbacks of the LIFT technique and, even more, can compete on an equal basis with the more conventional direct writing techniques.

The aim of this work is designing a sensitive chemiresistor sensor using a laser-processing technique (TP-LIFT), which considers cost and application requirements.

\section{Experimental}

\subsection{Laser-induced forward transfer}

The LIFT setup used in this work consists of the pulsed UV $\mathrm{XeCl}$ laser beam (308-nm emission wavelength, 30-ns pulse length, $1-\mathrm{Hz}$ repetition rate), which is guided and imaged with an optical system onto the donor substrate to transfer micropixels $(500 \times 500 \mu \mathrm{m})$ from the donor substrate to the receiving surface $[23,24]$. The laser fluence is varied from 100 to $500 \mathrm{~mJ} / \mathrm{cm}^{2}$. A computer-controlled $x y z$ translation stage allows the displacement of both donor and receptor substrates with respect to the laser beam. The donor and the receiver are kept in contact, while the laser irradiates the donor from the backside. For each laser pulse single micropixels are obtained. All experiments were performed under ambient pressure at room temperature. A scheme of the LIFT setup is shown in Fig. 1.

\subsection{Donor and receiving substrates}

The donor substrates were prepared by spin coating the TP (with a thickness of 100 and $300 \mathrm{~nm}$ ) onto fused silica plates (area $5 \mathrm{~cm}^{2}$, thickness $1 \mathrm{~mm}$ ) and then spin coating the SWCNT suspension on top of the TP layer. In Fig. 2, a scanning electron microscopy (SEM) image of the SWCNT donor film deposited onto a TP film is shown.
The TP was synthesized as described by Nagel et al. in [18], and was then prepared by spin coating from a solution in chlorobenzene and cyclohexanone $(1: 1, w / w)$. TP was chosen as DRL layer, due to the fact that the emission wavelength of the laser used in this work matches the absorption maxima of the TP.

Single-walled carbon nanotubes (SWCNT, HiPco) are purchased from Nanointegris (as a dry powder) and used without further purification.

In order to obtain SWCNT thin films, 10-mg CNT are dispersed in water $(15 \mathrm{ml})$ containing the nonionic surfactant Triton $X$ (with different polyoxyethylene chain lengths) $(100 \mathrm{mg})$, and the dispersion is bath sonicated for 40 min. The dispersion is then spin coated onto TP-coated fused silica plates under different conditions, i.e., rotation speed 1,500-2,500, ramp of 1,000-2,000 rpm, and spincoating duration of 30 and $60 \mathrm{~s}$. In order to remove any trace of solvent from the donor films, the spin-coated SWCNT were then left to dry on a hot plate for $2 \mathrm{~h}$ at $50{ }^{\circ} \mathrm{C}$.

As receiver substrates ordinary glass slides or fused silica plates are used which are cleaned in an ultrasonic bath with acetone, ethanol and distilled water prior to the experiments.

\subsection{Morphological and structural investigation}

The deposited features as well as the target prior to ablation were investigated by optical microscopy. The images were acquired with an Axiovert 200 Microscope coupled to a Carl Zeiss AxioCam MRm camera.

The distribution of the SWCNT on the donor and receiving surface was investigated by scanning electron microscopy (SEM). The images were obtained from top view SEM and were acquired with a Zeiss Supra VP55 FESEM operating at a voltage of $5 \mathrm{kV}$ and an in-lens detector.

Raman microscopy is used to check for any chemical changes in the structure of the SWCNT prior and after their deposition by LIFT. The Raman spectra of SWCNT are recorded on a Labram confocal Raman microscopy system
Fig. 1 Scheme of the LIFT setup
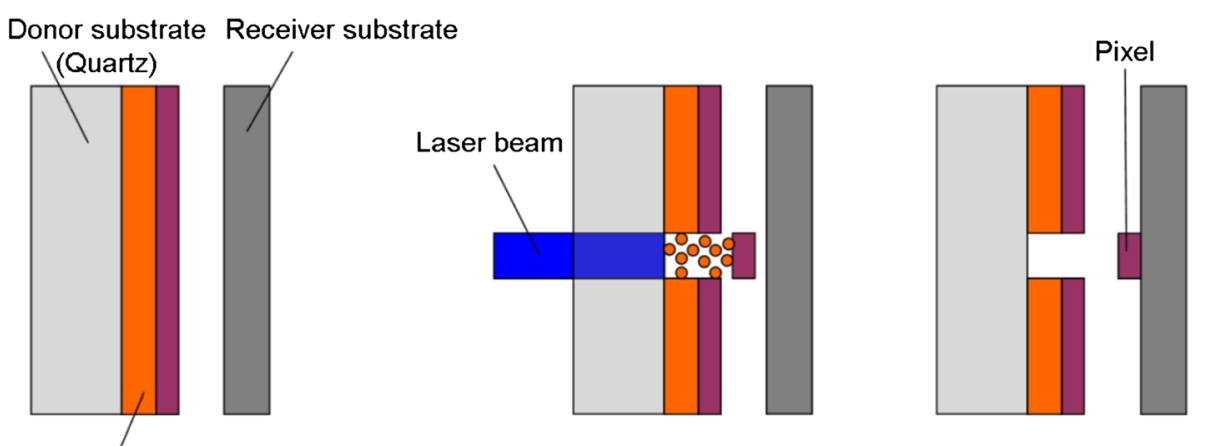

Dynamic release layer: triazene polymer 


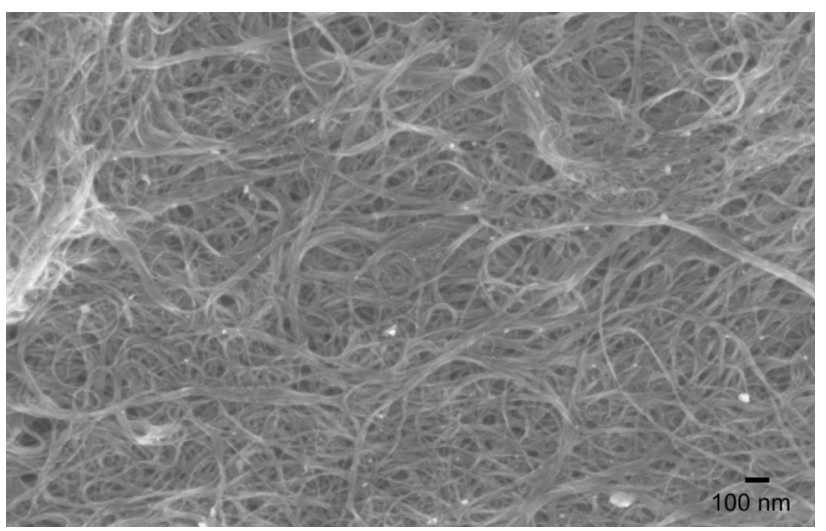

Fig. 2 SEM image of SWCNT deposited by spin coating onto a TPcoated quartz substrate

from Jobin-Yvon. The 633-nm line from a HeNe laser is used as an excitation source for the Raman spectra. The laser power at the sample surface is typically $20 \mathrm{~mW}$. For each measurement, the exposure time and the accumulation were $20 \mathrm{~s}$ and 5 times, respectively. Raman spectra are collected over the range of $200-3,000 \mathrm{~cm}^{-1}$. All spectra are recorded at room temperature.

\subsection{Sensitivity tests}

In order to carry out the sensitivity tests, the SWCNT pixels are transferred onto $\mathrm{Pt}$ electrodes. They are then mounted on a holder and electrically contacted with Ag paste. Resistance is monitored continuously with time by a computer-controlled setup using a Keithley 2,400 source meter. The holder is placed in a closed chamber with a constant gas supply and the baseline resistance is monitored. In order to test the sensors for their gas detection ability, 200-ppm ammonia was introduced in the test chamber using a chromatography syringe. Once the sensor reached saturation, the test chamber was purged with $\mathrm{N}_{2}$ gas until the signal recovered the original baseline value.

\section{Results and discussion}

\subsection{Morphological investigation of the SWCNT pixels}

The investigation of LIFT aiming applications such as the fabrication of chemiresistor sensors requires a systematic variation of different process parameters and an analysis of the resulting morphological and chemical features of the transferred materials.

The TP thickness is an important parameter for obtaining a regular, "clean" transfer. TP as DRL absorbs the laser radiation and subsequently decomposes into gaseous fragments, which are used to transform the energy into required mechanical push. In Fig. 3a, series of images depicting a SWCNT donor film deposited by spin coating onto a 300-nm-thick TP layer immediately after LIFT are shown. The laser fluences applied are in the range $100-500 \mathrm{~mJ} / \mathrm{cm}^{2}$. In Fig. 3a, the applied laser fluence of $100 \mathrm{~mJ} / \mathrm{cm}^{2}$ is too low to push forward the SWCNTs. In Fig. 3b, the formation of a "skin" which breaks due to the high pressure generated by the ablation of the TP can be observed. The "skin" represents undetached yet delaminated TP on the ablation spot with the SWCNT film on top [18]. Gas is released through the "skin"; however, no CNT are detached from the donor. In Fig. 3c-e, the laser fluence applied is too high, gas escapes from the sides of the pixel and spreads away the material around the ablated pixel. In all these images (c), (d), and (e) redeposited material, i.e., the pixel which is detached from the donor substrate, reaches the receiving substrate, and due to the high velocity is bounced back on the donor substrate [25]. The transfer of SWCNT with a 300-nm TP release layer and all applied conditions never results in a transfer of uniformly distributed pixels, i.e., never a CNT array, on the receiver substrate. Therefore, only 100-nm TP layer thicknesses are used. Similar observations have been reported for other systems, i.e., transfer of biomolecules (liposomes) in liquid phase [26]. The thickness of the TP together with the
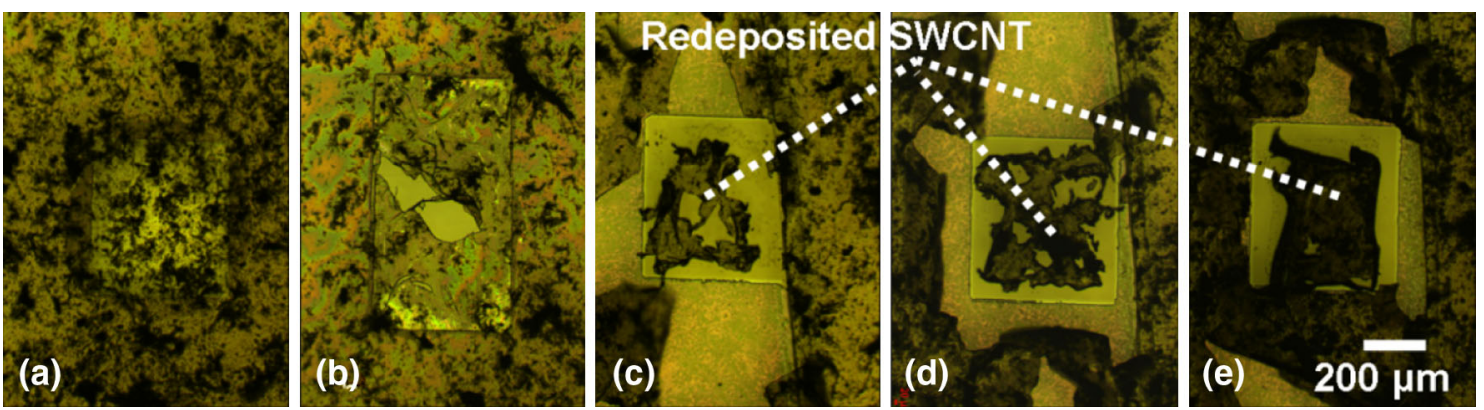

Fig. 3 Sequence of images from a SWCNT donor film deposited by spin coating onto a 300-nm-thick TP layer immediately after LIFT. Laser fluence applied a $100 \mathrm{~mJ} / \mathrm{cm}^{2}$, b $200 \mathrm{~mJ} / \mathrm{cm}^{2}$, c $300 \mathrm{~mJ} / \mathrm{cm}^{2}, \mathbf{d ~} 400 \mathrm{~mJ} / \mathrm{cm}^{2}$, and e $500 \mathrm{~mJ} / \mathrm{cm}^{2}$ 
Fig. 4 Optical microscopy images of a the donor (SWCNT on top of a $100 \mathrm{~nm}$ TP layer) prior to LIFT, $\mathbf{b}$ the donor immediately after LIFT, and c pixels deposited onto a glass substrates (laser fluence applied for the transfer $250 \mathrm{~mJ} / \mathrm{cm}^{2}$ )

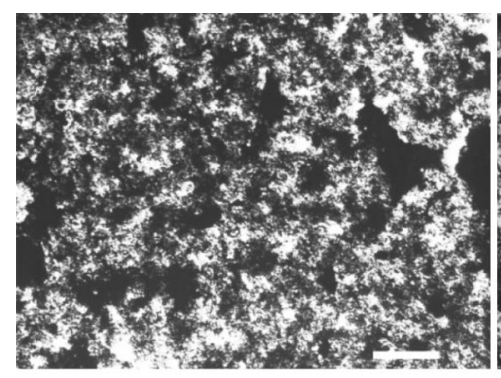

(a)
$50 \mu \mathrm{m}$

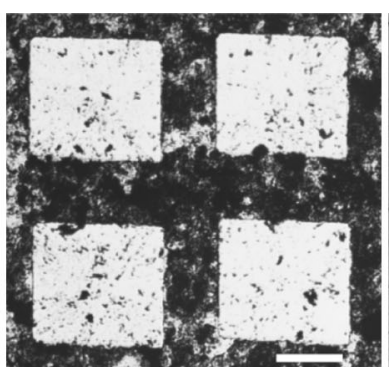

(b)

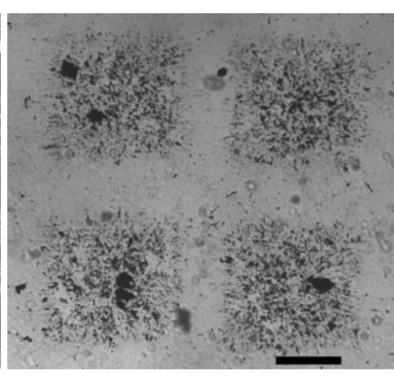

(c)

$200 \mu \mathrm{m}$ applied fluence is important because they determine the amount of released gas.

An optical microscopy image of the donor substrate prior to LIFT, the donor immediately after LIFT, and four pixels deposited onto a glass substrates at $250 \mathrm{~mJ} / \mathrm{cm}^{2}$ is shown in Fig. 4. With 100-nm TP interlayer it is possible to achieve a "clean" transfer, i.e., pixels with well-defined contours.

In addition, for the fabrication of a chemiresistor sensor it is important to control the laser fluence. The laser fluence is varied over a broad range, i.e., from conditions insufficient to break the donor layer to high irradiation fluences $\left(0.1-1 \mathrm{~J} / \mathrm{cm}^{2}\right)$, to optimize the shape of the transferred pixels without any chemical modification of the SWCNTs. The optimum laser fluence values are found in the range $200-400 \mathrm{~mJ} / \mathrm{cm}^{2}$.

\subsection{Structural characterization of the transferred SWCNT pixels}

The Raman spectra of the SWCNT in the donor substrate is shown in Fig. 5 in comparison to the Raman spectra acquired from a SWCNT pixel transferred at a laser fluence of $250 \mathrm{~mJ} / \mathrm{cm}^{2}$.

Both Raman spectra, i.e., of the reference as well as the pixel present the typical Raman bands of SWCNTs: weak bands located between 100 and $300 \mathrm{~cm}^{-1}$ attributed to the radial breathing mode (RBM) indicating the expansion and contraction of the tubes [27]. The peak near $1,330 \mathrm{~cm}^{-1}$ (D band) indicates disorder in the graphitic layer of the CNTs, while the peak near $1,580 \mathrm{~cm}^{-1}$ ( $\mathrm{G}$ band) is due to the tangential modes of the graphitic planes in the CNTs [27]. As it can seen in Fig. 5, the intensity of the D band is very low, which indicates a negligible presence of amorphous carbon impurities. The low intensity ratio of the two peaks $[\mathrm{I}(\mathrm{D}) / \mathrm{I}(\mathrm{G})]$ suggests that there is a low disorder and few defects in the nanotubes. Full width at half maxima (FWHM) of the D- and G-peaks was analyzed both for the reference sample as well as for the pixel transferred at $250 \mathrm{~mJ} / \mathrm{cm}^{-1}$ (shown in Table 1). This indicates that

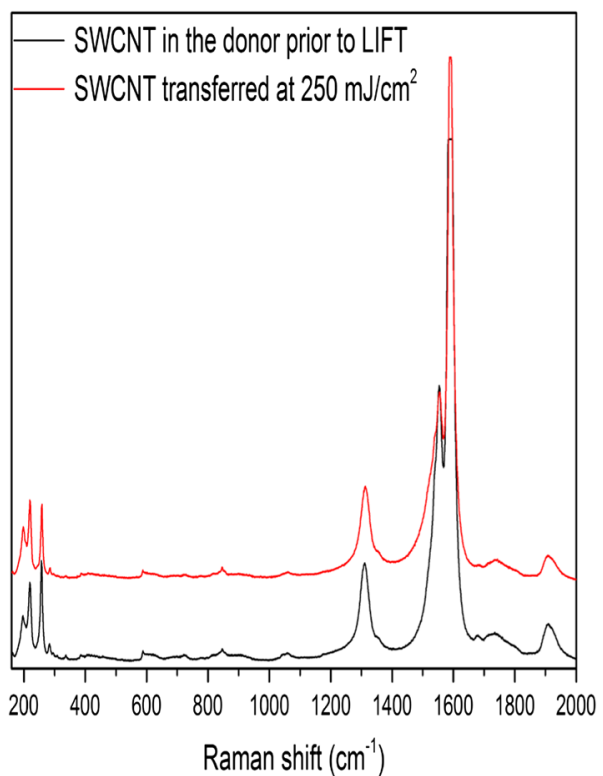

Fig. 5 Raman spectra comparing the initial donor material (SWCNT) and a SWCNT pixel transferred at $250 \mathrm{~mJ} / \mathrm{cm}^{2}$

Table 1 Comparison of the Raman spectral features of reference SWCNT, and SWCNT in the pixel transferred at $250 \mathrm{~mJ} / \mathrm{cm}^{2}$

\begin{tabular}{llll}
\hline Sample & $\mathrm{I}(\mathrm{D}) / \mathrm{I}(\mathrm{G})$ & \multicolumn{2}{l}{ FWHM $\left(\mathrm{cm}^{-1}\right)$} \\
\cline { 3 - 4 } & & $\mathrm{G}_{\text {band }}\left(\Delta \omega_{\mathrm{G}}\right)$ & $\mathrm{D}_{\text {band }}\left(\Delta \omega_{\mathrm{D}}\right)$ \\
\hline Reference & 0.098 & 21.25 & 35.8 \\
$\Phi=250 \mathrm{~mJ} / \mathrm{cm}^{2}$ & 0.112 & 20.14 & 40.63 \\
\hline
\end{tabular}

after LIFT, the SWCNT do not suffer any decomposition, and can be therefore further used in sensors applications.

\subsection{Sensitivity tests to ammonia}

For preparing functional SWCNT chemiresistor sensors, the first requirement is to test their electrical conductivity. These tests are carried out by transferring SWCNT pixels onto Pt electrodes and measuring their base resistance at 

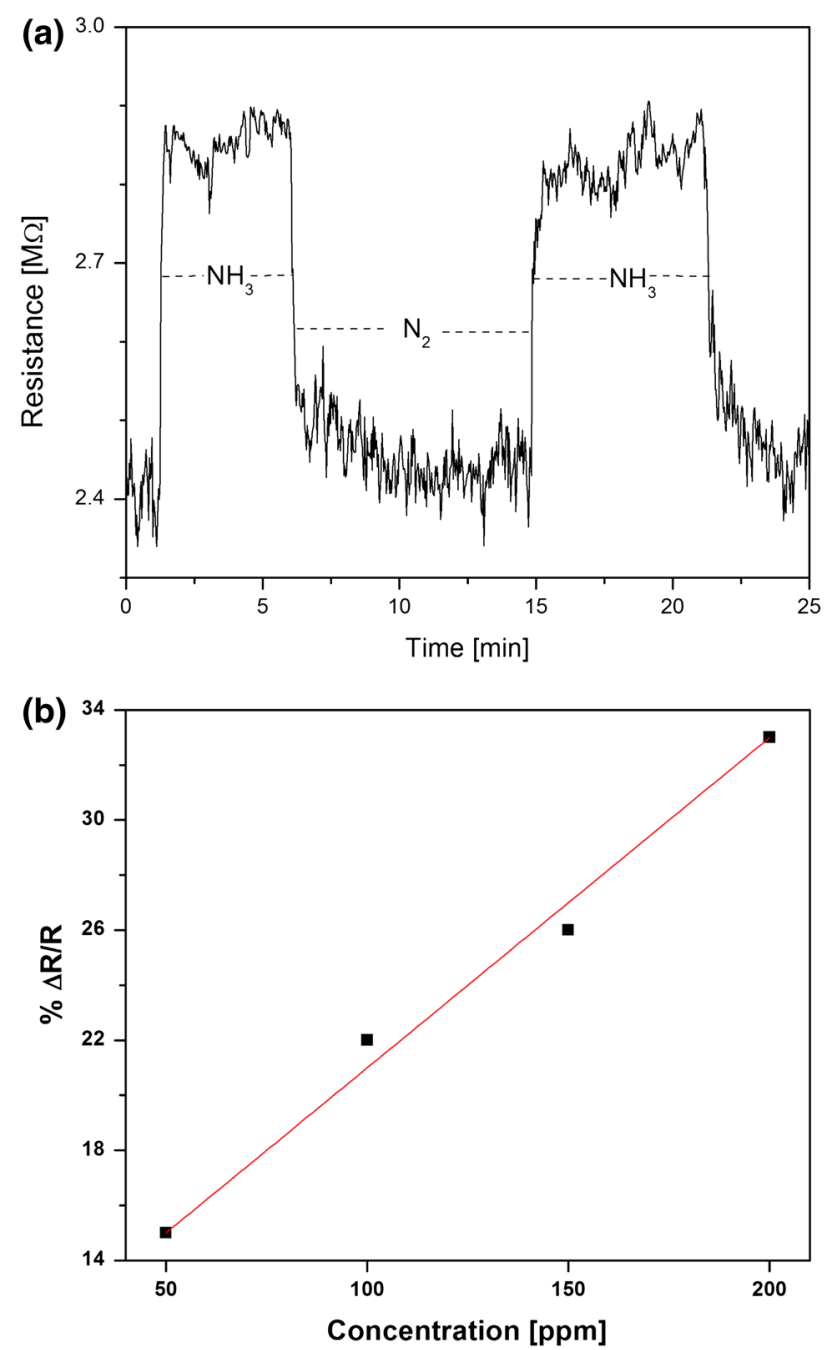

Fig. 6 a Sensor response upon exposure to $100 \mathrm{ppm}$ of ammonia. b Plot of sensor response as a function of ammonia concentration. \% $\Delta R / R$ for 5-min exposure to ammonia

room temperature. The resistance values of the SWCNT in the pixels were in the same range as in the donor substrates, i.e., $1-10 \mathrm{M} \Omega$.

In order to determine the sensing ability of the SWCNT transferred by LIFT onto Pt electrodes measurements with ppm concentrations of toxic compounds are carried out. The selected compound is ammonia, which is an electron-withdrawing vapor. The sensor is first introduced in the test chamber with a constant $\mathrm{N}_{2}$ gas flow. First, the sensor was found to have a stable baseline with minimum noise. Then, ammonia vapor with different concentrations, i.e., 50, 100, 150, and $200 \mathrm{ppm}$ is introduced in the test chamber. In Fig. 6a, an example of a typical sensor response to 100-ppm ammonia is shown. The LIFT-ed sensors show a repeatable response and recovery. The resistance increases sharply in the first minute, then the response begins to saturate in the next $10 \mathrm{~min}$. The test chamber is then purged using $\mathrm{N}_{2}$ gas and a spontaneous signal recovery is observed. The resistance spontaneously recovers up to $80 \%$ of its signal response within the first 1-2 min from the removal of the ammonia vapors. The sensitivities of the LIFT-ed SWCNT sensors to different ammonia concentrations are shown in Fig. 6b. The sensors show good repeatability and for all the experiments they exhibit a linear relationship with $\mathrm{NH}_{3}$ concentrations ranging from 50 to $200 \mathrm{ppm}$. The response of three LIFT-ed sensors to different ammonia concentrations was investigated to determine the variation in sensor response or the standard deviation. The standard deviation was estimated to be $\pm 7 \%$ for 150 and $200 \mathrm{ppm}$, and $\pm 5 \%$ for 50 and $100 \mathrm{ppm}$.

The response of the sensor tested to $\mathrm{NH}_{3}$ and its electrical behavior can be explained in terms of $p$-type characteristics. The adsorption of electron-withdrawing gas molecules in the CNTs can be attributed to the formation of an initial charge-transfer complex on the surface of the CNT bundles. This interaction is weak and reversible, and accounts for the spontaneous signal recovery measured for the sample.

\section{Conclusions}

The results shown above clearly reveal that SWCNT chemiresistor sensors can be fabricated by laser-induced forward transfer.

By investigating different process parameters reveals that both the TP thickness as well as the laser fluence applied for the transfer are important parameters for obtaining regular SWCNT patterns.

In order to prove the feasibility of LIFT for the fabrication of chemiresistor sensors, the SWCNT transferred by LIFT onto Pt electrodes have been measured upon 200-ppm concentration of ammonia. A sharp increase in resistance within the first 2 min of exposure has been noticed, with a spontaneous signal reversibility of up to $80 \%$ of the resistance within the first $1-2$ min.

However, to optimize the process, i.e., to reduce sensor to sensor variation due to the non-uniform deposition of the CNT on the TP-coated quartz slides, and to achieve a lower power consumption, alternative fabrication methods of the donor substrates are still required.

Acknowledgments Financial support from the Scientific Exchange Programme between Switzerland and the New Member States of the European Union (Sciex-NMS), through the Rectors Conference of the Swiss Universities (CRUS) project ALECSA “Application of laserinduced forward transfer for the fabrication of a flexible carbon nanotube sensor array" is gratefully acknowledged. 


\section{References}

1. M. Vrijheid, D. Martinez, S. Manzanares, P. Dadvand, A. Schembari, J. Rankin, M. Nieuwenhuijsen, Environ. Health Perspect. 119(5), 598-606 (2011)

2. T. Mattle, A. Hintennach, T. Lippert, A. Wokaun, Appl. Phys. A 110, 309-316 (2013)

3. Q. Cao, J.A. Rogers, Adv. Mater. 21, 29-53 (2009)

4. Y. Wang, S. Park, J.T.W. Yeow, A. Langner, F. Müller, Sens. Actuators B 149, 136-142 (2010)

5. Z. Wu, Z. Chen, X. Du, J.M. Logan, J. Sippel, M. Nikolou, K. Kamaras, J.R. Reynolds, D.B. Tanner, A.F. Hebard, A.G. Rinzler, Science 305, 1273-1276 (2004)

6. M.P. Garrett, I.N. Ivanov, R.A. Gerhardt, A.A. Puretzky, D.B. Geohegan, Appl. Phys. Lett. 97, 163105 (2010)

7. J.R.H. Shaw-Stewart, T. Mattle, T.K. Lippert, M. Nagel, F.A. Nuesch, A. Wokaun, J. Appl. Phys. 113, 043104 (2013)

8. M. Makrygianni, E. Verrelli, N. Boukos, S. Chatzandroulis, D. Tsoukalas, I. Zergioti, Appl. Phys. A 110, 559-563 (2013)

9. L. Rapp, F. Serein-Spirau, J.-P. Lère-Porte, A.P. Alloncle, P. Delaporte, F. Fages, C. Videlot-Ackermann, Org. Electron 13, 2035-2041 (2012)

10. A. Palla-Papavlu, C. Córdoba, A. Patrascioiu, J.M. FernándezPradas, J.L. Morenza, P. Serra, Appl. Phys. A 110, 751-755 (2013)

11. B. Hopp, T. Smausz, N. Kresz, N. Barna, Z. Bor, L. Kolozsvári, D.B. Chrisey, A. Szabó, A. Nógrádi, Tissue Eng. 11(11-12), 1817-1823 (2005)

12. C. Unger, M. Gruene, L. Koch, J. Koch, B.N. Chichkov, Appl. Phys. A 103, 271-277 (2011)

13. C.B. Arnold, P. Serra, A. Piqué, Mater. Res. Soc. Bull. 32, 23-31 (2007)
14. P. Serra, M. Colina, J.M. Fernández-Pradas, L. Sevilla, J.L. Morenza, Appl. Phys. Lett. 85(9), 1639-1641 (2004)

15. R. Fardel, M. Nagel, F. Nüesch, T. Lippert, A. Wokaun, Appl. Phys. Lett. 91, 061103 (2007)

16. S.K. Chang-Jian, J.R. Ho, J.-W.J. Cheng, C.K. Sung, Nanotechnology 17, 1184-1187 (2006)

17. C. Boutopoulos, C. Pandis, K. Giannakopoulos, P. Pissis, I. Zergioti, Appl. Phys. Lett. 96, 041104 (2010)

18. M. Nagel, R. Fardel, P. Feurer, M. Häberli, F.A. Nüesch, T. Lippert, A. Wokaun, Appl. Phys. A 92, 781-789 (2008)

19. T. Lippert, Adv. Polym. Sci. 168, 51-246 (2004)

20. T. Lippert, T. Dickinson, Chem. Rev. 103, $453-485$ (2003)

21. M.S. Brown, N.T. Kattamis, C.B. Arnold, J. Appl. Phys. 107, $083103(2010)$

22. N.T. Kattamis, P.E. Purnick, R. Weiss, C.B. Arnold, Appl. Phys. Lett. 91, 171120 (2007)

23. D. Cannatà, M. Benetti, F. Di Pietrantonio, E. Verona, A. PallaPapavlu, V. Dinca, M. Dinescu, T. Lippert, Sens. Actuators B 173, 32-39 (2012)

24. F. Di Pietrantonio, M. Benetti, D. Cannatà, E. Verona, A. PallaPapavlu, V. Dinca, M. Dinescu, T. Mattle, T. Lippert, Sens. Actuators B 174, 158-167 (2012)

25. A. Palla-Papavlu, V. Dinca, C. Luculescu, J. Shaw-Stewart, M. Nagel, T. Lippert, M. Dinescu, J. Opt. 12, 124014 (2010)

26. A. Palla-Papavlu, V. Dinca, M. Dinescu, F. Di Pietrantonio, D. Cannatà, M. Benetti, E. Verona, Appl. Phys. A 105, 651-659 (2011)

27. H. Chen, M.R. Golder, F. Wang, R. Jasti, A.K. Swan, Carbon 67, 203-213 (2014) 